\title{
Population Pharmacokinetics of Linezolid: A Systematic Review
}

\author{
Yan Qin ${ }^{1}$, Li-li Zhang ${ }^{2}$, Yan-rong Ye $^{2}$, Yue-ting Chen ${ }^{2}$, and Zheng Jiao ${ }^{3}$ \\ ${ }^{1}$ Shanghai Chest Hospital \\ ${ }^{2}$ Affiliation not available \\ ${ }^{3}$ Shanghai Jiao Tong University Affiliated Chest Hospital
}

February 10, 2022

\begin{abstract}
Background Linezolid is often used for the infections caused by drug-resistant Gram-positive bacteria. Recent studies suggested that large between-subject variability (BSV) and within-subject variability could alter drug pharmacokinetics (PK) during linezolid therapy due to pathophysiological changes. Objective The review synthesized information on linezolid population PK studies and summarized the significant covariates that influence the PKs of linezolid. Methods A literature search was performed from PubMed, Web of Science, and Embase from their inception to 30 September 2021. Published studies were included if they contained data analyzing linezolid PK parameters in humans using a population approach with a nonlinear mixed-effects model. Results Twenty-five studies were included in adults and five studies in pediatric patients. One- and two-compartment models were the commonly used structural models for linezolid. Body size [weight, lean body weight, and body surface area], creatinine clearance (CLcr), and age significantly influenced linezolid PK. The median clearance (CL) values (range) in infants $[0.128 \mathrm{~L} / \mathrm{h} / \mathrm{kg}(0.121-0.135)]$ and children $[0.107 \mathrm{~L} / \mathrm{h} / \mathrm{kg}(0.088-0.151)]$ were higher than in adults [0.098 L/h/kg (0.044-0.237)] For patients with severe renal impairment (CLcr [?] $30 \mathrm{~mL} / \mathrm{min})$, the CL was $37.2 \%(15.2-55.3 \%)$ lower than in patients with normal renal function. Conclusion Linezolid's optimal dosage could be adjusted based on the patient's body size, renal function, and age. More studies are needed to explore the exact mechanism of elimination of linezolid and evaluate PK characteristics in pediatric patients.
\end{abstract}

\section{Population Pharmacokinetics of Linezolid: A Systematic Review}

Yan Qin $^{1,2^{*}}$, Li-Li Zhang ${ }^{3^{*}}$, Yan-Rong Ye ${ }^{2^{*}}$, Yue-Ting Chen ${ }^{1}$, Zheng Jiao ${ }^{1}$

${ }^{1}$ Department of Pharmacy, Shanghai Chest Hospital, Shanghai Jiao Tong University, Shanghai, China.

${ }^{2}$ Department of Pharmacy, Zhongshan Hospital, Fudan University, Shanghai, China

${ }^{3}$ Department of Pharmacy and Purchasing Management, the 900th Hospital of PLA joint logistics support force, Fuzhou, China.

Running heading: population pharmacokinetics of linezolid

Correspondence:

Zheng Jiao, Professor

Email: jiaozhen@online.sh.cn

ORCID: 0000-0001-7999-7162

* Yan Qin, Li-Li Zhang and Yan-Rong Ye contributed equally to this work 


\title{
Acknowledgments
}

The authors would like to sincerely thank Dr Jason A. Roberts from Burns, Trauma and Critical Care Research Centre, The University of Queensland, Australia for providing details about the research and active discussions on the coding. We would like to thank Chen-yu Wang MPharm from Shanghai Chest Hospital; and Ye Zou Pharmacist from Department of Pharmacy, Zhongshan Hospital, Fudan University, China for their critical comments.

\begin{abstract}
Background Linezolid is often used for the infections caused by drug-resistant Gram-positive bacteria. Recent studies suggested that large between-subject variability (BSV) and within-subject variability could alter drug pharmacokinetics (PK) during linezolid therapy due to pathophysiological changes.

Objective The review synthesized information on linezolid population PK studies and summarized the significant covariates that influence the PKs of linezolid.

Methods A literature search was performed from PubMed, Web of Science, and Embase from their inception to 30 September 2021. Published studies were included if they contained data analyzing linezolid PK parameters in humans using a population approach with a nonlinear mixed-effects model.

Results Twenty-five studies were included in adults and five studies in pediatric patients. One- and twocompartment models were the commonly used structural models for linezolid. Body size [weight, lean body weight, and body surface area], creatinine clearance (CLcr), and age significantly influenced linezolid PK. The median clearance (CL) values (range) in infants $[0.128 \mathrm{~L} / \mathrm{h} / \mathrm{kg}(0.121-0.135)]$ and children $[0.107$ $\mathrm{L} / \mathrm{h} / \mathrm{kg}(0.088-0.151)]$ were higher than in adults $[0.098 \mathrm{~L} / \mathrm{h} / \mathrm{kg}(0.044-0.237)]$. For patients with severe renal impairment (CLcr [?] $30 \mathrm{~mL} / \mathrm{min}$ ), the CL was $37.2 \%$ (15.2-55.3\%) lower than in patients with normal renal function.

Conclusion Linezolid's optimal dosage could be adjusted based on the patient's body size, renal function, and age. More studies are needed to explore the exact mechanism of elimination of linezolid and evaluate PK characteristics in pediatric patients.
\end{abstract}

\section{Key words}

linezolid, individualized drug therapy, nonlinear mixed-effects model, population pharmacokinetics

\section{Introduction}

Linezolid, an oxazolidinone antibiotics, plays an essential role against drug-resistant Gram-positive bacteria, such as methicillin-resistantStaphylococcus aureus, vancomycin-resistantEnterococci, coagulase-negative Staphylococci, penicillin-insensitive Streptococcus pneumoniae, andmultidrug-resistant and extensively drug-resistant Mycobacterium tuberculosis [1-4]. It is approved for treating infections induced by susceptible strains of designated microorganisms, such as bacteremia, nosocomial pneumonia, skin and soft tissue infections, and community-acquired pneumonia $[1,5]$.

Linezolid bioavailability is approximately $100 \%$, and no dosage adjustment is necessary when switching from intravenous to oral therapy. Food does not affect linezolid absorption [6]. Linezolid plasma protein binding is approximately $31 \%$, and its volume of distribution is approximately $40-50 \mathrm{~L}$ in adults $[7,8]$. Linezolid is primarily metabolized in the liver through oxidation of the morpholine ring, producing two major inactive metabolites: PNU142586 and PNU142300. Linezolid inhibits its own metabolism. Linezolid clearance can be inhibited to 51.3-85.5\% of its initial value over time, resulting in nonlinear elimination [9-11]. Approximately $30 \%$ of linezolid is excreted unchanged in the urine through the kidneys. Non-renal clearance is around $65 \%$ of total linezolid clearance $[12,13]$. Linezolid has a time-dependent antibacterial activity with mild to 
moderate post-antibiotic effects. The efficacy pharmacodynamic targets are the ratio of the area under the concentration-time curve over 24 hours $\left(\mathrm{AUC}_{0-24 \mathrm{~h}}\right)$ to the minimum inhibitory concentration (MIC) of 80$120\left(\mathrm{AUC}_{0-24 \mathrm{~h}} / \mathrm{MIC}=80-120\right.$, ideal 100$)$ and the percentage of time the drug concentration remains greater than the MIC (T\% > MIC) for at least $85 \%$ of the dosing interval, ideal $100 \%$, at a steady-state [14].

Recent studies suggest a large between-subject variability (BSV) for linezolid with a remarkable percentage of subtherapeutic levels in critically ill patients [15-18]. Inadequate exposure can lead to therapy failure and bacterial resistance. Among the adverse effects of linezolid, reversible thrombocytopenia has attracted the most attention and is associated with increased exposure and a longer duration of therapy [8, 1921]. Furthermore, sepsis, diabetes, total body weight (TBW), renal function, renal replacement therapies, and liver function could influence linezolid clearance [11, 22-25]. Therefore, it is necessary to perform an individualized drug therapy considering the pharmacokinetics (PK) of linezolid and factors that affect drug exposure.

The population PK ( PPK) approach is a powerful tool for evaluating factors that influence PK in target patients and helps physicians identify high-risk patient subgroups for therapy failure. The PPK model can optimize individual doses through Bayesian forecasting. Moreover, Monte Carlo simulations based on the PPK model allow for the prediction of covariate effects in the target population and estimation of the probabilities of target attainment (PTA). Extensive PPK studies for linezolid have been performed in various populations; however, data regarding linezolid PPK modeling have not been summarized so far. This review provides synthetic information on linezolid PPK studies and aims to summarize the significant covariates that influence the pharmacokinetics of linezolid and identify any issues that need further exploration.

\section{Methods}

\subsection{Search strategy}

The review focused on the PPK of linezolid. The literature was systemically searched from PubMed, Web of Science, and Embase from its inception to 30 September 2021 according to the Preferred Reporting Items for Systematic Reviews and Meta-Analyses (PRISMA-2020) guidelines [26]. The following search terms were used: 'linezolid' or 'Zyvox' or 'PNU-100766' or 'U-100766' or 'oxazolidinone' and 'population pharmacokinetic*' or 'pharmacokinetic model*' or 'nonlinear mixed effect model' or 'NONMEM' or 'WINNONMIX' or 'ADAPT' or 'P-PHARM' or 'nlmixed' or 'NLME' or 'MONOLIX.' Detailed search strategies were listed in the electronic Supplementary Table S1. In addition, reference lists of the selected articles were screened to identify other relevant papers. Two independent authors conducted the title and abstract screening followed by the full-text screening using EndNote (version 9.2; Thomson Scientific, Box Hill, Victoria, Australia). Discrepancies were resolved by consulting a senior investigator.

Identified studies included in this review should meet the following criteria: (1) study population: human studies (healthy subjects or patients); (2) treatment: linezolid administered by oral route or intravenous infusion; (3) modeling approach: a parametric non-linear mixed effect modeling approach. Studies were excluded if (1) they were reviews or methodology articles; (2) data overlapped with later published articles; (3) studies lacked the necessary model information; and (4) studies written in a language other than English.

\subsection{Data Extraction}

The following information was extracted from eligible articles by two independent authors: (1) demographic data of the population (e.g., country, age, body size, gender), (2) study characteristics (e.g., dosage regimens, bioanalytical methods, sample numbers, sampling schedule), and (3) PPK characteristics such as analysis software and algorithm, structural models, covariates, covariate screening method, PK parameters and formula, BSV, and residual unexplained variability (RUV). 


\subsection{Quality Assessment}

The quality of the included studies was assessed using a 35 item checklist (Table S2) considered essential for the reporting of clinical PK studies [27, 28]. The items proposed in the checklist were categorized into five parts based on relevance to a traditional PPK research report: title/abstract, background, methods, results, and discussion/conclusion. One point was given for each item if the information contained in the identified study met the criteria. Otherwise, the item was given 0 points. Compliance was used to evaluate the quality of each PPK study, which was calculated using the following equation:

compliance $(\%)=($ sum of items reported / sum of all items $) \times 100 \%$

\subsection{Study comparison}

The characteristics of the studies and PPK analyses were summarized in a tabular format for comparison. One thousand virtual patients were simulated and their concentration-time profiles were plotted according to the published PPK models in each identified study. Virtual patients were defined as follows: neonates (4 $\mathrm{kg}, 14$ days) taking $40 \mathrm{mg}$ of linezolid every $8 \mathrm{~h}$ (q8h), infants (10 kg, 1 year) taking $100 \mathrm{mg}$ of linezolid q8h, children $(25 \mathrm{~kg}$, 7 years) taking $250 \mathrm{mg}$ of linezolid q8h, adults (70 kg, 40 years) taking $600 \mathrm{mg}$ of linezolid q12h, and elderly (70 kg, 80 years) taking $600 \mathrm{mg}$ of linezolid q12h. A steady-state was assumed if a patient received multiple doses of linezolid. All virtual patients were set as men. The simulation was run using NONMEM (version 7.5; ICON Development Solutions, Ellicott City, MD, USA). The concentration-time profiles were plotted using $\mathrm{R}$ software (version 4.1.1; http://www.r-project.org/).

The impact of identified covariates on clearance (CL) in each study was summarized and compared using the forest map, conducted using the forestplot package (version 1.9; https://gforge.se/packages/) within the $\mathrm{R}$ packages. Continuous covariates were scaled to the same range for comparison. If continuous covariates were identified in only one study, the minimum and maximum values of the covariates in this study were used. For binary covariates such as dialysis, 0 was for dialysis patients during linezolid treatment and 1 for patients without dialysis. The minimum and maximum CL values were calculated according to the range of the identified covariates in each study. The reference value of CL was normalized to the median covariate values in each study. The effect of each covariate on CL was expressed as the percentage of the CL range divided by the CL reference value. A change in CL of less than $80 \%$ or more than $125 \%$ was considered clinically significant [29].

\section{Results}

\subsection{Study identification}

A total of 851 studies were identified: 354 from PubMed, 107 from Embase, and 390 from Web of Science. An additional study was obtained by checking the reference lists of the included articles. Forty-four studies were eligible for full-text screening after duplicate removal and title and/or abstract selection. Fourteen studies were excluded due to missing PPK parameters, not being written in English, using a nonparametric method to establish PPK models or duplicate data reported in other articles. Finally, 30 studies were included in this review for further analysis. The PRISMA flow diagram of study identification is presented in Fig.1.

\subsection{Evaluation of the literature}

The quality results of the PPK studies are shown in Table 1. Compliance with each study ranged from 68.6 to $97.1 \%$, with a median value of $85.7 \%$. Most studies did not report the route of linezolid administration in the tile/abstract section, co-medications, food interactions, methods for handling missing data in the method section, and the schematics of the final model in the results section. 


\subsection{Study characteristics}

All studies were published between 2003 and 2021. The characteristics of the included PPK studies are listed in Table 2. Six studies were conducted in China [17, 30-34], six in Japan [24, 35-39], four in Germany [22, 40-42], and two in Italy [4, 43]. Another 12 studies were conducted in the US, UK, Brazil, Australia, Spain, France, Korea, Greece, Canada, and South Africa [18, 25, 44-49]. Most of the studies were single-center and seven were multicenter studies $[7,11,33,38,47,50,51]$. Sparse samplings were employed in 19 studies including pre-dose and 1-2 h post-dose $[4,17,22,25,32-34,36-39,44-50]$. The number of participants in each study ranged from 9 to 603, and 19 studies included less than 50. In most studies, subjects were patients with Gram-positive bacterial infections, with comorbidity of sepsis, burns, liver impairment, and renal impairment with or without renal replacement therapy. Two studies were conducted in tuberculosis patients [46, 48]. Only one study consisted of both patients and healthy volunteers [11]. Among the included studies, five studies were conducted in pediatrics and another 25 studies were conducted in adults.

In most studies, linezolid was administered at a dose of $600 \mathrm{mg} \mathrm{q} 12 \mathrm{~h}$ to adults (Table 2). The dose regimens for pediatric patients were $10 \mathrm{mg} / \mathrm{kg}$ q8h or q12h or q24h. Plasma linezolid concentrations were determined by liquid chromatography with ultraviolet detection $(n=23)$ or mass spectrometry $(n=7)$. The lowest limit of quantification ranged from 0.001 to $0.78 \mathrm{mg} / \mathrm{L}$.

\subsection{Population pharmacokinetic characteristics}

Most PPK models were developed with NONMEM software, with the exception of five studies that were developed by ADAPT II [44], Monolix [50], Pumas [49], and Phoenix NLME [32, 34]. The algorithms used mainly were first-order conditional estimation (FOCE) or FOCE with $\eta-\epsilon$ interaction. Eighteen studies described linezolid PPK as a one-compartment model and 11 as a two-compartment model. Only one study used a three-compartment model. First-order elimination was reported in 27 studies. One study suggested that linezolid followed the elimination of time-dependent autoinhibition [11]. Another two studies reported that parallel first-order renal and Michaelis-Menten (MM) nonrenal elimination was superior to a simple first-order or MM elimination model [42, 44] (Table 2).

\subsubsection{Clearance and volume of distribution}

The median CLs in infants and children were higher than in adults, and the median CL in neonates was much lower than in other populations. The estimated median CL of linezolid was $0.042 \mathrm{~L} / \mathrm{h} / \mathrm{kg}$ for neonates $(4 \mathrm{~kg}$, 14 days), $0.128 \mathrm{~L} / \mathrm{h} / \mathrm{kg}$ (range: $0.121-0.135$ ) for infants (10kg, 1 year), $0.107 \mathrm{~L} / \mathrm{h} / \mathrm{kg}$ (range: $0.088-0.151$ ) for children (25kg, 7 years), and $0.098 \mathrm{~L} / \mathrm{h} / \mathrm{kg}$ (range: 0.044-0.237) for adults (70 kg 40 years). Additionally, no significant ethnic differences were shown in the PK profile of linezolid. The PK parameters were close among Caucasians, Asians, and Africans. The median volumes of distribution (V) per kilogram in neonates and children were higher than in adults and infants. The median $\mathrm{V}$ was $0.675 \mathrm{~L} / \mathrm{kg}$ for neonates (4kg, 14 days), $0.5 \mathrm{~L} / \mathrm{kg}$ (range: $0.41-0.59)$ for infants $(10 \mathrm{~kg}, 1$ year), $0.784 \mathrm{~L} / \mathrm{kg}$ (range: $0.592-1.36)$ for children $(25 \mathrm{~kg}, 7$ years), and $0.594 \mathrm{~L} / \mathrm{kg}$ (range: $0.197-2.99)$ for adults (70 kg, 40 years).

\subsubsection{Random effects}

BSV was described by an exponential model in all studies. The median coefficient of variation (CV) for BSV was as follows: CL, $46.6 \%$ (range: 23.2- 66.9\%), and V, 33.9\% (range: 10.9-142.0\%). RUV was described by proportional models in 14 studies [7, 11, 24,31,32, 34, 36, 37, 39, 42, 46, 47, 49, 50], additive models in two studies [25, 35], and combined proportional and additive models in 14 studies $[4,17,18,22,30,33,38,40$, $41,43-45,48,51$ ]. The proportional RUV ranged from $2.5 \%$ to $102 \%$ (median $17.5 \%$ ) and the additive RUV ranged from $0.007 \mathrm{mg} / \mathrm{L}$ to $1.9 \mathrm{mg} / \mathrm{L}$ (median $0.26 \mathrm{mg} / \mathrm{L}$ ) among all studies. Only one study reported an inter-occasion variability of $23.0 \%$ in CL and V.

\subsubsection{Covariate screening}

Most studies were intended to identify covariates to explain the BSV of linezolid PK, except for three studies, in which no covariates were investigated due to the limited included subjects. A stepwise method including 
forward inclusion and backward elimination was the most frequently used method for covariate screening. All covariates tested and identified on CL and V are summarized in Table S3. The most influential covariates were body size, renal function, and age. The impact of each covariate on CL is shown in Fig.2.

Body sizes, including total body weight (TBW) and body surface area (BSA), were reported in $16(53.3 \%)$ studies, all of which except one study had a significant influence on CL with a change of larger than $20 \%$, as shown in Fig.2. Furthermore, the effect of renal function (CLcr or eGFR) was reported in 13 (43.3\%) studies, and nine of them showed a change of CL greater than 20\%. For patients with severe renal impairment (CLcr [?] $30 \mathrm{~mL} / \mathrm{min})$, the CL was $37.2 \%$ (15.2-55.3\%) lower than that in patients with normal renal function.

Five studies investigated the influence of postnatal age on CL in pediatric patients, and two of them showed that the change was greater than $20 \%$. Furthermore, CL was found to increase with increasing postnatal age. The linezolid concentration-time profile in neonates (4 kg, 14 days) was significantly higher than in infants and children with the same dose per weight, as shown in Fig.3.

Five of the 25 studies found that age was associated with linezolid CL in adult patients, and the influence of age on CL was greater than $20 \%$ in three reported studies. Meanwhile, four studies indicated that age was negatively correlated with CL. Elderly patients $(70 \mathrm{~kg}, 80$ years) showed considerably higher PK profiles than adults (70 kg, 40 years) in three studies $[4,39,50]$.

Seventeen studies investigated the impact of liver function on CL, seven of which found that CL in patients with liver dysfunction was reduced by 36.4-71.5\% [36, 37, 44, 53, 58-60]. Liver cirrhosis, liver transplant, or liver resection, and laboratory tests such as alanine aminotransferase (ALT), aspartate aminotransferase (AST), maximal liver function capacity (LiMAx), and prothrombin (PTA) were identified as significant covariates on CL.

Body sizes, including TBW, lean body weight (LBW), and BSA, were the most reported covariates influencing V. Additionally, peritonitis was reported to affect V (Table 3). No other covariates were identified.

\subsubsection{Model evaluation}

All models were assessed by an internal evaluation. Diagnostic plots, bootstraps, and visual predictive checks were the most commonly used methods. A normalized prediction distribution error (NPDE) was adopted in three studies $[30,33,47]$. Furthermore, only one study was evaluated using an independent dataset and showed acceptable predictability [51]. External validation is recommended to evaluate the predictive performance of the PPK model prior to clinical implementation.

\subsubsection{Model informed precision dosing}

Sixteen studies conducted a model-based simulation to propose dosing regimens to achieve the [?] $80 \%$ or 90\% PTA, defined as AUC/MIC 80-120 [17, 24, 25, 30, 32-35, 42, 46, 48, 49, 51]. Six studies also adopted T\% > MIC being $100 \%$ or $85 \%$ as a supplementary target $[17,24,42,47,49,51]$. In addition, three other studies used only linezolid trough concentration $\left(\mathrm{C}_{0}\right)$ as the target index.

Linezolid dosage regimens were proposed based on the developed models. For neonates, $8 \mathrm{mg} / \mathrm{kg}$ (q8h) for an MIC of $1 \mathrm{mg} / \mathrm{L}$ and $12 \mathrm{mg} / \mathrm{kg}$ (q8h) for an MIC of $2 \mathrm{mg} / \mathrm{L}$ were required to reach PTA > 90\% [25]. Dosage regimens ranging from 10 to $20 \mathrm{mg} / \mathrm{kg}$ of body weight $\mathrm{q} 6 \mathrm{~h}$ or q $8 \mathrm{~h}$ were recommended for children $[30,34]$. For obese patients with body weights over $100 \mathrm{~kg}, 600 \mathrm{mg}$ q $8 \mathrm{~h}$ or more was required to achieve PTAs of [?] $90 \%$ for a MIC of $2 \mathrm{mg} / \mathrm{L}$ [42, 49]. A linezolid dosing regimen for adult patients with renal impairment was recommended, which ranged from 300 to $1,200 \mathrm{mg}$ q12 h based on renal function [4, 24, $32,33,35,37$ ] (Table 4). Dosing regimens of $200 \mathrm{mg}$ or $300 \mathrm{mg}$ (q12h) were recommended for patients with liver impairment [32,35]. Some studies also found that if MICs were [?] $4 \mathrm{mg} / \mathrm{L}$, low PTAs were obtained even with increased linezolid dosing [33, 42, 49]. 


\section{Discussion}

Linezolid is an essential therapeutic choice against multidrug-resistant Gram-positive bacteria. Unfortunately, linezolid's large variabilities make individualized dosing regimens necessary [52]. Several PPK studies of linezolid have been conducted to identify the source of variability among different populations. Our systematic review is the first to summarize the PPK of linezolid to facilitate the optimal pharmacotherapy of linezolid.

Weight was the most significant covariate that affected linezolid PK in pediatric patients. Total clearance increased with increasing weight in most studies. Infants and children showed higher CL per weight than adults, which could be attributed to a higher body fat/lean mass ratio, decreased kidney blood flow, and lower total body water in adults. Two studies reported that postnatal age was also a significant covariate that influenced linezolid CL in pediatric patients [25, 39], which probably reflects the maturation of the liver and kidney, which are essential for linezolid PK.

For adult patients, nine studies reported that weight had a significant influence on CL $[7,22,36,38,40$, $44,45,47,49]$, with eight studies indicating a considerable change greater than $20 \%$. For patients with a bodyweight of $40 \mathrm{~kg}$, the CL of linezolid decreased by $34.3 \%$ (15.8-46.6\%) compared to patients with a normal body weight of $70 \mathrm{~kg}$, indicating that a reduced dose may be needed in patients with low body weight. On the contrary, for obese patients with a body weight of $120 \mathrm{~kg}$, the CL of linezolid increased by $54.3 \%$ (42.2-82.9\%) compared to the CL of $70 \mathrm{~kg}$ patients. Therefore, obese patients may be at higher risk of not achieving the pharmacodynamic target at the standard dose of linezolid, and an increased dose may be appropriate.

Both the classic PK analysis and the PPK approach suggested that renal clearance accounted for about $30 \%$ of the total clearance of linezolid $[8,12,44]$. Fourteen population PK studies in this review identified $\mathrm{CL}_{\mathrm{CR}}$ as a covariate on CL $[4,11,22,23,30,32,33,35-38,43,44,51]$. Furthermore, serum creatinine instead of CLcr was selected as a covariate on CL in one study of neonates [25] and four studies conducted in adults $[31,42,43,49]$. Patients with renal replacement therapy (such as hemodialysis, hemodiafiltration, and hemofiltration) had a higher clearance of linezolid [22, 24, 40]. Clearance varied with the dialysis method, the afferent flow rate, and the efferent flow rate $[22,24]$. In studies in which $\mathrm{CL}_{\mathrm{CR}}$ or creatinine was not identified as a covariate on CL, most study subjects had a normal renal function or mild renal impairment $[17,25,34,37,47]$. Linezolid dosing regimens need to be adjusted according to renal impairment.

Five studies indicated that linezolid PK was influenced by age in adult patients $[4,7,17,38,47]$, and $80 \%$ of these studies found that linezolid CL decreased with age. CL in elderly patients (70 kg, 80 years) was reduced by $20.82-79.66 \%$ compared to that of adult patients (70 kg, 40 years), possibly due to the decrease in renal or liver function as a result of aging. Previous studies showed that reduced ROS production could be caused by aging, but the exact mechanism of aging remains unexplained, which requires further investigation [7].

Linezolid is mainly metabolized in the liver. Liver function (ALT, AST) influenced linezolid clearance in several studies. A notable decrease in CL (about 50\%-60\%) was observed in patients with liver transplantation/resection or patients with severe liver cirrhosis (Child-Pugh C) $[35,36]$. The decrease is possibly due to fibrosis, damage to the lobular structure, altered enzyme expression, and reduced blood flow. These changes result in less transport of drugs and oxygen to hepatocytes [53].

Several classic PK studies demonstrated that clearance after multiple doses was smaller than that of a single dose or the first dose, which could be explained by time-dependent autoinhibition of linezolid elimination in the liver [54-57]. Linezolid could inhibit the enzyme activity of the mitochondrial oxidative respiratory chain at therapeutic levels, resulting in less ATP produced from oxidative phosphorylation and reduced linezolid metabolisms $[9,12,58,59]$. However, many included PPK studies in this review reported that linezolid showed first-order elimination. Only one study reported time-dependent autoinhibition, and two described parallel first-order and MM elimination. The results could be attributed to the treatment being not long enough to produce time-dependent autoinhibition. 


\section{Limitations}

Our systematic review has the following limitations. Models developed from nonparametric methods were omitted. Only studies published in English were selected. Since this review aimed to identify critical covariates that influence linezolid PKs and compare linezolid PKs among different age groups, PK/PD models were not summarized.

\section{Conclusion}

This review summarized key aspects related to linezolid PPK for clinical application and future research. Weight, renal function, and age significantly influenced linezolid CL, and linezolid dose should be individualized. Meanwhile, considering the high BSV and RUV of linezolid PKs, therapeutic drug monitoring may be helpful in maintaining optimal linezolid exposure. More PPK studies are needed in pediatric patients, along with larger sample sizes. The exact mechanism of elimination of linezolid needs to be explored to facilitate the design of optimal dosing regimens.

\section{Declarations}

Conflict of Interest Disclosure Yan Qin, Li-li Zhang, Yan-rong Ye, Yue-ting Chen, and Zheng Jiao declare they have no conflicts of interest.

Ethics Approval Statement Not applicable.

Funding Statement Not applicable.

Patient Consent Statement Not applicable.

Consent for Publication Not applicable.

Availability of Data and Material The datasets generated during and/or analysed during the current study are available from the corresponding author on reasonable request.

Code Availability Not applicable.

Author contributions Yan Qin: Investigation, Data curation, Write- Original draft, Writing-Reviewing and Editing, Visualization;Li-li Zhang: Investigation, Write- Original draft, Writing- Reviewing and Editing; Yan-rong Ye: Writing- Reviewing and Editing, Supervision; Yue-ting Chen: Soft, Validation;Zheng Jiao: Conceptualization, Methodology, Writing- Reviewing and Editing, Supervision.

\section{Reference:}

1. Hashemian, S.M.R., Farhadi, T., Ganjparvar, M. Linezolid: a review of its properties, function, and use in critical care. Drug. Des. Devel. Ther . $2018,12,1759-1767$.

2. Zhang, X., Falagas, M.E., Vardakas, K.Z., Wang, R., Qin, R., Wang, J., Liu, Y. Systematic review and meta-analysis of the efficacy and safety of therapy with linezolid containing regimens in the treatment of multidrug-resistant and extensively drug-resistant tuberculosis. J. Thorac. Dis . 2015 , 7(4),603-615.

3. Faella, F., Pagliano, P., Fusco, U., Attanasio, V., Conte, M. Combined treatment with ceftriaxone and linezolid of pneumococcal meningitis: a case series including penicillin-resistant strains.Clin. Microbiol. Infect . $2006,12(4), 391-394$.

4. Crass, R.L., Cojutti, P.G., Pai, M.P., Pea, F. Reappraisal of Linezolid Dosing in Renal Impairment To Improve Safety.Antimicrob. Agents. Chemother . 2019, 63(8).

5. Zahedi, B.A., Rahbar, M., Yousefi, M., Asgharzadeh, M., Samadi, K. H. Linezolid: a promising option in the treatment of Gram-positives.J Antimicrob Chemother . 2017, 72(2),354-364. 
6. Welshman, I.R., Sisson, T.A., Jungbluth, G.L., Stalke,r D.J., Hopkins, N.K. Linezolid absolute bioavailability and the effect of food on oral bioavailability. Biopharm. Drug. Dispos . 2001, 22(3),91-97.

7. Abe, S., Chiba, K., Cirincione, B., Grasela, T.H., Ito, K., Suwa, T. Population pharmacokinetic analysis of linezolid in patients with infectious disease: application to lower body weight and elderly patients. $J$. Clin. Pharmacol . 2009, 49(9),1071-1078.

8. Boak, L.M., Rayner, C.R., Grayson, M.L., Paterson, D.L., Spelman, D., Khumra, S., Capitano, B., Forrest, A., Li, J., Nation, R.L., Bulitta, J.B. Clinical population pharmacokinetics and toxicodynamics of linezolid. Antimicrob. Agents. Chemother . 2014, 58(4),2334-2343.

9. Plock, N., Buerger, C., Joukhadar, C., Kljucar, S., Kloft, C. Does linezolid inhibit its own metabolism? Population pharmacokinetics as a tool to explain the observed nonlinearity in both healthy volunteers and septic patients. Drug. Metab. Dispos . 2007 , 35(10),1816-1823.

10. Bhalodi, A.A., Papasavas, P.K., Tishler, D.S., Nicolau, D.P., Kuti, J.L. Pharmacokinetics of intravenous linezolid in moderately to morbidly obese adults. Antimicrob. Agents. Chemother . 2013, 57(3),1144-1149.

11. Minichmayr, I.K., Schaeftlein, A., Kuti, J.L., Zeitlinger, M., Kloft, C. Clinical Determinants of Target Non-Attainment of Linezolid in Plasma and Interstitial Space Fluid: A Pooled Population Pharmacokinetic Analysis with Focus on Critically Ill Patients. Clin. Pharmacokinet . 2017 , 56(6),617-633.

12. Slatter, J.G., Stalker, D.J., Feenstra, K.L., Welshman, I.R., Bruss, J.B., Sams, J.P., Johnson, M.G., Sanders, P.E., Hauer, M.J., Fagerness, P.E., Stryd, R.P., Peng, G.W., Shobe, E.M. Pharmacokinetics, metabolism, and excretion of linezolid following an oral dose of $[(14) \mathrm{C}]$ linezolid to healthy human subjects. Drug. Metab. Dispos . 2001, 29(8),1136-1145.

13. MacGowan, A.P. Pharmacokinetic and pharmacodynamic profile of linezolid in healthy volunteers and patients with Gram-positive infections. J. Antimicrob. Chemother . 2003, 51 Suppl 2,ii17-25.

14. Rayner, C.R., Forrest, A., Meagher, A.K., Birmingham, M.C., Schentag, J.J. Clinical pharmacodynamics of linezolid in seriously ill patients treated in a compassionate use programme. Clin Pharmacokinet $\mathbf{2 0 0 3}$ , 42(15),1411-1423.

15. Sazdanovic, P., Jankovic, S.M., Kostic, M., Dimitrijevic, A., Stefanovic, S. Pharmacokinetics of linezolid in critically ill patients.Expert. Opin. Drug. Metab. Toxicol . 2016 , 12(6),595-600.

16. Roger, C., Muller, L., Wallis, S.C., Louart, B., Saissi, G., Lipman, J., Lefrant, J.Y., Roberts, J.A. Population pharmacokinetics of linezolid in critically ill patients on renal replacement therapy: comparison of equal doses in continuous venovenous haemofiltration and continuous venovenous haemodiafiltration. $J$. Antimicrob. Chemother . 2016 , 71(2),464-470.

17. Dong, H., Xie, J., Wang, T., Chen, L., Zeng, X., Sun, J., Wang, X., Dong, Y. Pharmacokinetic/pharmacodynamic evaluation of linezolid for the treatment of staphylococcal infections in critically ill patients.Int. J. Antimicrob. Agents . 2016 , 48(3),259-264.

18. Luque, S., Grau, S., Alvarez-Lerma, F., Ferrandez, O., Campillo, N., Horcajada, J.P., Basas, M., Lipman, J., Roberts, J.A. Plasma and cerebrospinal fluid concentrations of linezolid in neurosurgical critically ill patients with proven or suspected central nervous system infections. Int. J. Antimicrob. Agents . 2014, 44(5),409-415.

19. Hanai, Y., Matsuo, K., Ogawa, M., Higashi, A., Kimura, I., Hirayama, S., Kosugi, T., Nishizawa, K., Yoshio, T. A retrospective study of the risk factors for linezolid-induced thrombocytopenia and anemia. $J$. Infect. Chemother . 2016 , 22(8),536-542.

20. Nukui, Y., Hatakeyama, S., Okamoto, K., Yamamoto, T., Hisaka, A., Suzuki, H., Yata, N., Yotsuyanagi, H., Moriya, K. High plasma linezolid concentration and impaired renal function affect development of linezolid-induced thrombocytopenia. J. Antimicrob. Chemother .2013, 68(9),2128-2133. 
21. Tsuji, Y., Hiraki, Y., Matsumoto, K., Mizoguchi, A., Kobayashi, T., Sadoh, S., Morita, K., Kamimura, H., Karube, Y. Thrombocytopenia and anemia caused by a persistent high linezolid concentration in patients with renal dysfunction. J. Infect. Chemother . 2011, 17(1),70-75.

22. Wicha, S.G., Frey, O.R., Roehr, A.C., Pratschke, J., Stockmann, M., Alraish, R., Wuensch, T., Kaffarnik, M. Linezolid in liver failure: exploring the value of the maximal liver function capacity (LiMAx) test in a pharmacokinetic pilot study. Int. J. Antimicrob. Agents .2017, 50(4),557-563.

23. Cojutti, P., Pai, M.P., Pea, F. Population Pharmacokinetics and Dosing Considerations for the Use of Linezolid in Overweight and Obese Adult Patients. Clin. Pharmacokinet . 2018 , 57(8),989-1000.

24. Ide, T., Takesue, Y., Ikawa, K., Morikawa, N., Ueda, T., Takahashi, Y., Nakajima, K., Takeda, K., Nishi, S. Population pharmacokinetics/pharmacodynamics of linezolid in sepsis patients with and without continuous renal replacement therapy. Int. J. Antimicrob. Agents . 2018, 51(5),745-751.

25. Thibault, C., Kassir, N., Goyer, I., Theoret, Y., Litalien, C., Moussa, A., Ovetchkine, P., Autmizguine J. Population Pharmacokinetics of Intravenous Linezolid in Premature Infants. Pediatr. Infect. Dis. J . $2019,38(1), 82-88$.

26. Yepes-Nunez, J.J., Urrutia, G., Romero-Garcia, M., Alonso-Fernandez, S. The PRISMA 2020 statement: an updated guideline for reporting systematic reviews. Rev. Esp. Cardiol (Engl Ed) . 2021 , 74(9),790-799.

27. Kanji, S., Hayes, M., Ling, A., Shamseer, L., Chant, C., Edwards, D.J., Edwards, S., Ensom, M.H., Foster, D.R., Hardy, B., Kiser, T.H., la Porte, C., Roberts, J.A., Shulman, R., Walker, S., Zelenitsky, S., Moher D. Reporting Guidelines for Clinical Pharmacokinetic Studies: The ClinPK Statement. Clin. Pharmacokinet . 2015, 54(7),783-795.

28. Jamsen, K.M., McLeay, S.C., Barras, M.A., Green, B. Reporting a population pharmacokineticpharmacodynamic study: a journal's perspective. Clin. Pharmacokinet . 2014, 53(2),111-122.

29. Christians, U., Klawitter, J., Clavijo, C.F. Bioequivalence testing of immunosuppressants: concepts and misconceptions. Kidney. Int. Suppl . 2010 (115),S1-7.

30. Li, S.C., Ye, Q., Xu, H., Zhang, L., Wang, Y. Population Pharmacokinetics and Dosing Optimization of Linezolid in Pediatric Patients. Antimicrob Agents Chemother . 2019 , 63(4).

31. Wang, D., Zheng, X., Yang, Y., Chen, X. Population pharmacokinetic analysis of linezolid in patients with different types of shock: Effect of platelet count. Exp. Ther. Med . 2019, 18(3),1786-1792.

32. Zhang, S.H., Zhu, Z.Y., Chen, Z., Li, Y., Zou, Y., Yan, M., Xu, Y., Wang, F., Liu, M.Z., Zhang, M., Zhang, B.K. Population Pharmacokinetics and Dosage Optimization of Linezolid in Patients with Liver Dysfunction.Antimicrob. Agents. Chemother . 2020, 64(6).

33. Wang, X., Wang, Y., Yao, F., Chen, S., Hou, Y., Zheng, Z., Luo, J., Qiu, B., Li, Z., Wang, Y., Wu, Z., Lan, J., Chen, C. Pharmacokinetics of Linezolid Dose Adjustment for Creatinine Clearance in Critically Ill Patients: A Multicenter, Prospective, Open-Label, Observational Study.Drug. Des. Devel. Ther . 2021 , 15,2129-2141.

34. Yang, M., Zhao, L., Wang, X., Sun, C., Gao, H., Wang, X., Qian, S. Population Pharmacokinetics and Dosage Optimization of Linezolid in Critically Ill Pediatric Patients. Antimicrob. Agents. Chemother .2021

35. Sasaki, T., Takane, H., Ogawa, K., Isagawa, S., Hirota, T., Higuchi, S., Horii, T., Otsubo, K., Ieiri, I. Population pharmacokinetic and pharmacodynamic analysis of linezolid and a hematologic side effect, thrombocytopenia, in Japanese patients. Antimicrob. Agents. Chemother . 2011 , 55(5),1867-1873.

36. Tsuji, Y., Yukawa, E., Hiraki, Y., Matsumoto, K., Mizoguchi, A., Morita, K., Kamimura, H., Karube, Y., To, H. Population pharmacokinetic analysis of linezolid in low body weight patients with renal dysfunction. J. Clin. Pharmacol . 2013, 53(9),967-973. 
37. Matsumoto, K., Shigemi, A., Takeshita, A., Watanabe, E., Yokoyama, Y., Ikawa, K., Morikawa, N., Takeda, Y. Analysis of thrombocytopenic effects and population pharmacokinetics of linezolid: a dosage strategy according to the trough concentration target and renal function in adult patients. Int. J. Antimicrob. Agents . 2014, 44(3),242-247.

38. Tsuji, Y., Holford, N.H.G., Kasai, H., Ogami, C., Heo, Y.A., Higashi, Y., Mizoguchi, A., To, H., Yamamoto, Y. Population pharmacokinetics and pharmacodynamics of linezolid-induced thrombocytopenia in hospitalized patients. Br. J. Clin. Pharmacol . 2017, 83(8),1758-1772.

39. Ogami, C., Tsuji, Y., To, H., Yamamoto, Y. Pharmacokinetics, toxicity and clinical efficacy of linezolid in Japanese pediatric patients. J. Infect. Chemother . 2019 , 25(12),979-986.

40. Swoboda, S., Ober, M.C., Lichtenstern, C., Saleh, S., Schwenger, V., Sonntag, H.G., Haefeli, W.E., Hempel, G., Hoppe-Tichy, T., Weigand, M.A. Pharmacokinetics of linezolid in septic patients with and without extended dialysis. Eur. J. Clin. Pharmacol . 2010, 66(3),291-298.

41. Taubert, M., Zoller, M., Maier, B., Frechen, S., Scharf, C., Holdt, L.M., Frey, L., Vogeser, M., Fuhr, U., Zander, J. Predictors of Inadequate Linezolid Concentrations after Standard Dosing in Critically Ill Patients. Antimicrob. Agents. Chemother . 2016 , 60(9),5254-5261.

42. Ehmann, L., Simon, P., Busse, D., Petroff, D., Dorn, C., Huisinga, W., Dietrich, A., Zeitlinger, M., Wrigge, H., Kloft, C. Risk of target non-attainment in obese compared to non-obese patients in calculated linezolid therapy. Clin. Microbiol. Infect . 2020 , 26(9),1222-1228.

43. Allegra, S., Di Paolo, A., Cusato, J., Fatiguso, G., Arrigoni, E., Danesi, R., Corcione, S., D'Avolio, A. A Common mdr1 Gene Polymorphism is Associated With Changes in Linezolid Clearance. Ther. Drug. Monit . 2018, 40(5),602-609.

44. Meagher, A.K., Forrest, A., Rayner, C.R., Birmingham, M.C., Schentag, J.J. Population pharmacokinetics of linezolid in patients treated in a compassionate-use program. Antimicrob. Agents. Chemother. $2003,47(2), 548-553$.

45. Whitehouse, T., Cepeda, J.A., Shulman, R., Aarons, L., Nalda-Molina, R., Tobin, C., MacGowan, A., Shaw, S., Kibbler, C., Singer, M., Wilson, A.P. Pharmacokinetic studies of linezolid and teicoplanin in the critically ill. J. Antimicrob. Chemother . 2005, 55(3),333-340.

46. Strydom, N., Gupta, S.V., Fox, W.S., Via, L.E., Bang, H., Lee, M., Eum, S., Shim, T., Barry, C.E., Zimmerman, M., Dartois, V., Savic, R.M. Tuberculosis drugs' distribution and emergence of resistance in patient's lung lesions: A mechanistic model and tool for regimen and dose optimization. PLoS. Med . 2019 , 16(4),e1002773.

47. Xie, F., Mantzarlis, K., Malliotakis, P., Koulouras, V., Degroote, S., Koulenti, D., Blot, S., Boussery, K., Van Bocxlaer, J., Colin, P. Pharmacokinetic evaluation of linezolid administered intravenously in obese patients with pneumonia. J. Antimicrob. Chemother .2019, 74(3),667-674.

48. Garcia-Prats, A.J., Schaaf, H.S., Draper, H.R., Garcia-Cremades, M., Winckler, J., Wiesner, L., Hesseling, A.C., Savic, R.M. Pharmacokinetics, optimal dosing, and safety of linezolid in children with multidrugresistant tuberculosis: Combined data from two prospective observational studies. PLoS. Med . 2019, $16(4), \mathrm{e} 1002789$.

49. Blackman, A.L., Jarugula, P., Nicolau, D.P., Chui, S.H., Joshi, M., Heil, E.L., Gopalakrishnan, M. Evaluation of Linezolid Pharmacokinetics in Critically Ill Obese Patients with Severe Skin and Soft Tissue Infections. Antimicrob. Agents. Chemother . 2021, 65(2).

50. Alghamdi, W.A., Al-Shaer, M.H., An, G., Alsultan, A., Kipiani, M., Barbakadze, K., Mikiashvili, L., Ashkin, D., Griffith, D.E., Cegielski, J.P., Kempker, R.R., Peloquin, C.A. Population Pharmacokinetics of Linezolid in Tuberculosis Patients: Dosing Regimen Simulation and Target Attainment Analysis. Antimicrob. Agents. Chemother .2020, 64(10). 
51. Soraluce, A., Barrasa, H., Asin-Prieto, E., Sanchez-Izquierdo, J.A., Maynar, J., Isla, A., RodriguezGascon, A. Novel Population Pharmacokinetic Model for Linezolid in Critically Ill Patients and Evaluation of the Adequacy of the Current Dosing Recommendation.Pharmaceutics . 2020 , 12(1).

52. Wicha, S.G., Märtson, A.G., Nielsen, E.I., Koch, B.C.P., Friberg, L.E., Alffenaar, J.W., Minichmayr, I.K. From Therapeutic Drug Monitoring to Model-Informed Precision Dosing for Antibiotics. Clinical. Pharmacology \&3 Therapeutics . 2021, 109(4),928-941.

53. Le Couteur, D.G., Fraser, R., Hilmer, S., Rivory, L.P., McLean, A.J. The hepatic sinusoid in aging and cirrhosis: effects on hepatic substrate disposition and drug clearance. Clin. Pharmacokinet .2005 , $44(2), 187-200$.

54. Stalker, D.J., Jungbluth, G.L., Hopkins, N.K., Batts, D.H. Pharmacokinetics and tolerance of single- and multiple-dose oral or intravenous linezolid, an oxazolidinone antibiotic, in healthy volunteers. J. Antimicrob. Chemother . 2003 , 51(5),1239-1246.

55. Dehghanyar, P., Burger, C., Zeitlinger, M., Islinger, F., Kovar, F., Muller, M., Kloft, C., Joukhadar, C. Penetration of linezolid into soft tissues of healthy volunteers after single and multiple doses.Antimicrob. Agents. Chemother . 2005 , 49(6),2367-2371.

56. Buerger, C., Plock, N., Dehghanyar, P., Joukhadar, C., Kloft, C. Pharmacokinetics of unbound linezolid in plasma and tissue interstitium of critically ill patients after multiple dosing using microdialysis.Antimicrob. Agents. Chemother . 2006 , 50(7),2455-2463.

57. Eslam, R.B., Burian, A., Vila, G., Sauermann, R., Hammer, A., Frenzel, D., Minichmayr, I.K., Kloft, C., Matzneller, P., Oesterreicher, Z., Zeitlinger, M. Target site pharmacokinetics of linezolid after single and multiple doses in diabetic patients with soft tissue infection. J. Clin. Pharmacol . 2014, 54(9),1058-1062.

58. De Vriese, A.S., Coster, R.V., Smet, J., Seneca, S., Lovering, A., Van Haute, L.L., Vanopdenbosch, L.J., Martin, J.J., Groote, C.C., Vandecasteele, S., Boelaert, J.R. Linezolid-induced inhibition of mitochondrial protein synthesis. Clin. Infect. Dis .2006 , 42(8),1111-1117.

59. Wynalda, M.A., Hauer, M.J., Wienkers, L.C. Oxidation of the novel oxazolidinone antibiotic linezolid in human liver microsomes.Drug. Metab. Dispos . 2000 , 28(9),1014-1017.

\section{Hosted file}

Figure legend.docx available at https://authorea.com/users/459885/articles/556000-populationpharmacokinetics-of-linezolid-a-systematic-review 

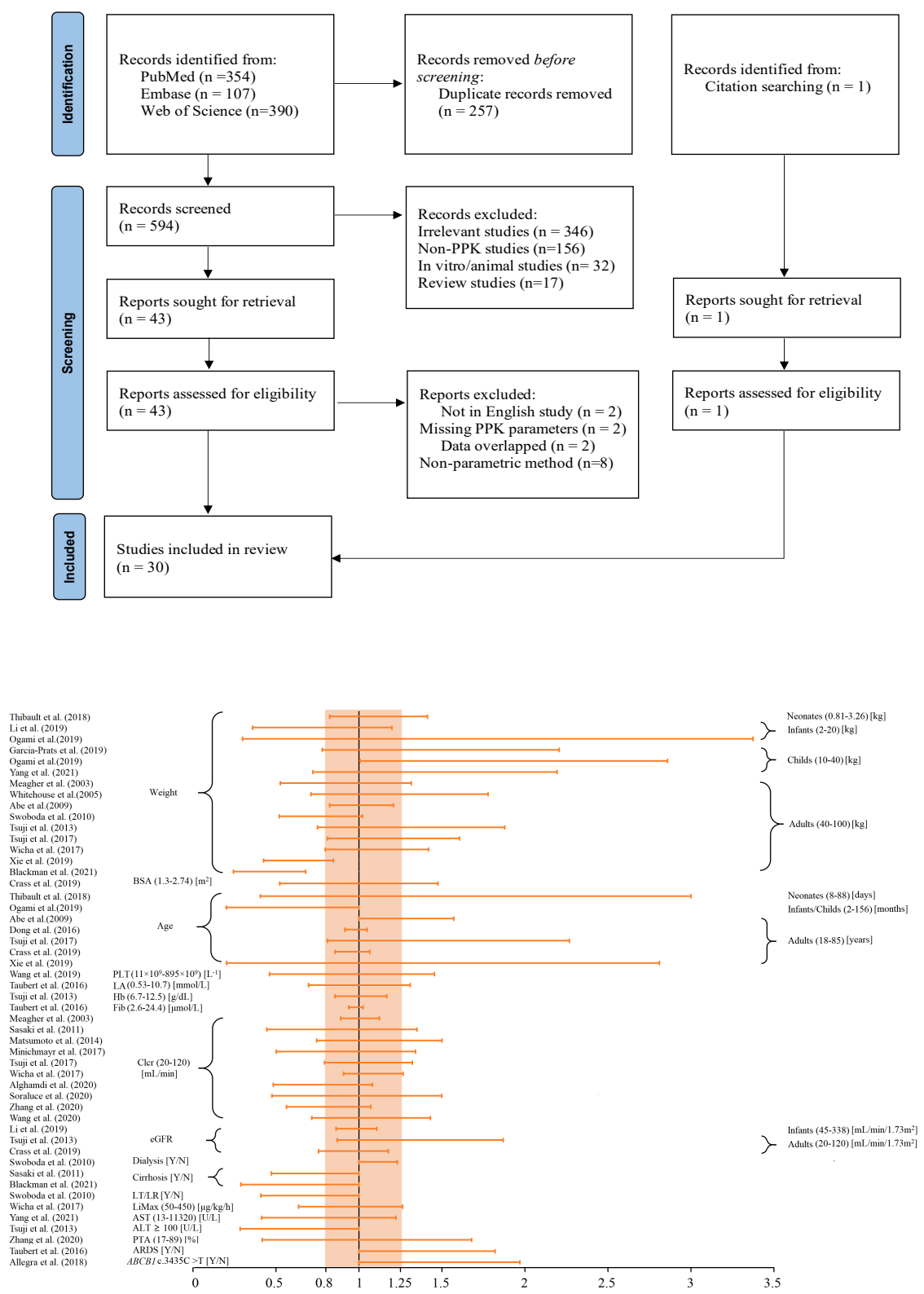


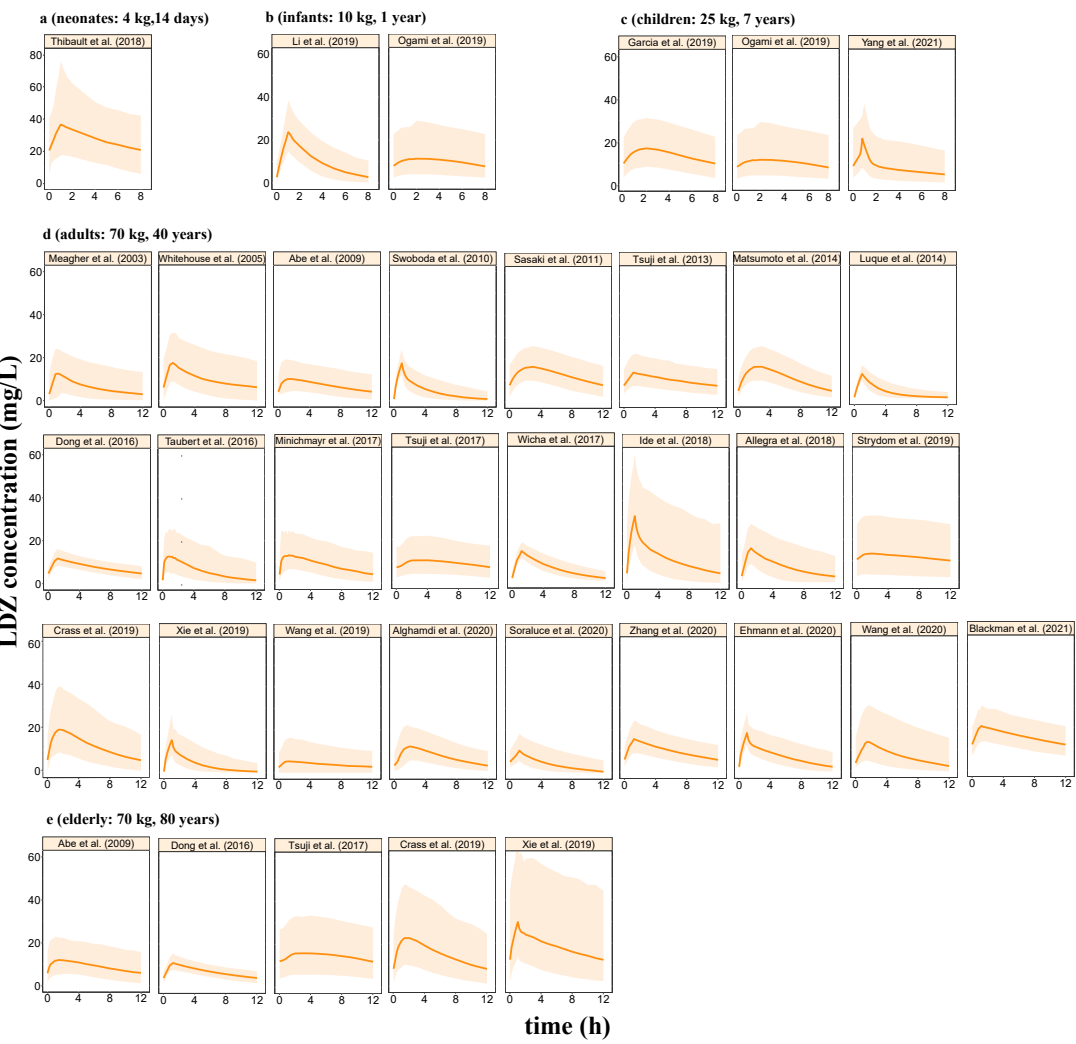

\section{Hosted file}

Table 1.pdf available at https://authorea.com/users/459885/articles/556000-populationpharmacokinetics-of-linezolid-a-systematic-review

\section{Hosted file}

Table 2.pdf available at https://authorea.com/users/459885/articles/556000-populationpharmacokinetics-of-linezolid-a-systematic-review

\section{Hosted file}

Table 3.pdf available at https://authorea.com/users/459885/articles/556000-populationpharmacokinetics-of-linezolid-a-systematic-review

\section{Hosted file}

Table 4.docx available at https://authorea.com/users/459885/articles/556000-populationpharmacokinetics-of-linezolid-a-systematic-review 